\title{
PBIBDs from weakly divisible nearrings and related codes
}

\author{
Anna Benini
}

\begin{abstract}
In [5] the authors are able to give a method for the construction of a family of partially balanced incomplete block designs from a special class of wd-nearrings ( $w d$-designs). In this paper the wd-design incidence matrix and the connected row and column codes are studied. The parameters of two special classes of wd-designs and those of the related row and column codes are calculated.
\end{abstract}

\section{Introduction}

In [10] a general method to construct partially balanced incomplete block designs on the set $X$ is given, starting from a transitive permutation group $H$ on $X$ with an intransitive subgroup $S$.

Obviously, from such a general method no formulae arise for the design parameters computation. A possible way to obtain the computability of the parameters is to link the action of $H$ to the action of some operation defined on $X$, making $X$ an algebraic structure. This could be an efficient tool to work with. Following such a line, in [5], a class of partially balanced incomplete block designs (wd-designs) is constructed and formulae for their parameters are found.

In this paper the incidence matrix of the above designs and the related row and column codes are studied. In more detail:

In Section 2 the basic definitions related to partially balanced incomplete block designs (PBIBDs) can be found and a frame of the whole construction, based on $[3,4,5]$, is given.

In Section 3 the incidence matrix of the constructed $P B I B D$ s is studied.

In Section 4 the parameters of the row and column codes related to the above incidence matrix are found.

In Section 5 two special classes of $P B I B D$ s are considered, for which all the parameters can be easily calculated.

\section{Partially Balanced Incomplete Block Designs}

A Partially Balanced Incomplete Block Design is a complex structure: a block design and an association scheme are defined together on the same set and they fit. We give the basic definitions here.

\footnotetext{
${ }^{0} \mathrm{MSC}$ : primary 05B05, secondary 05E30, 94B25

Keywords : block designs, association schemes, cyclic codes

Work carried out on behalf of Italian M.I.U.R.
} 
Definition 2.1 An incidence structure $(X, \mathcal{B} \subseteq \mathcal{P}(X))$ is called block design (or tactical configuration) if all the blocks have the same size $k$ and all the elements have the same replication number $r$, which means they occur in the same number $r$ of blocks. A block design is said to be incomplete (IB-design) if at least one of its blocks is a proper subset of $X$. The numbers $(v, b, r, k)$, where $v$ and $b$ are the cardinality of $X$ and $\mathcal{B}$ respectively, are called the parameters of the design. We know that they are not independent, because $v r=b k$ holds.

For more information on design theory we refer to [7].

Definition 2.2 An association scheme with $m$ associate classes on a finite set $X$ is a family of $m$ symmetric and antireflexive binary relations $R_{1}, \ldots, R_{m}$ on $X$ such that:

(i) any two distinct elements of $X$ are $i$ th associates for exactly one value of $i=1, \ldots, m$;

(ii) for all $i=1, \ldots, m$ and $x \in X$, there are exactly $n_{i}$ distinct elements $y \in X$ so that $(x, y) \in R_{i}$;

(iii) for all $i, j, k=1, \ldots, m$, if $(x, y) \in R_{k}$, the number $p_{i j}^{k}$ of $z \in X$ so that $(x, z) \in R_{i}$ and $(y, z) \in R_{j}$ is a constant depending on $i, j, k$ but not on the particular choice of $x$ and $y$.

For further information about association schemes see [2].

Definition 2.3 A tactical configuration $(X, \mathcal{B})$ with an association scheme on $X$ is called partially balanced incomplete block design $(P B I B D)$ if there are positive integers $\lambda_{i}, i=1, \ldots, m$, such that, if $x, y \in X$ are any two $i$ th associate elements, then $x, y$ occur together in exactly $\lambda_{i}$ blocks of $\mathcal{B}$.

Thus a $P B I B D$ has the tactical configuration parameters $v, b, r$ and $k$, the association scheme parameters $n_{i}$ and $p_{i j}^{k}$, and the partial balance parameters $\lambda_{i}$, in addition. We refer to [13] for more information on $P B I B D$ s.

\section{$2.1 \quad P B I B D$ s from wd-nearrings}

A left nearring is an algebraic structure $N=(S,+, *)$ such that $(S,+)$ is an additive group, $(S, *)$ is a multiplicative semigroup, and the left distributive low holds (see $[8,14]$ ). A weakly divisible nearring (wd-nearring) is a left nearring $N$ in which at least one of the equations $a x=b$ or $b x=a$ has a solution in $N$, for all $a, b \in N$ (see [6]).

From $[3,4]$, we know that a class of wd-nearrings is constructible starting from a pair $(G, \Phi)$, where $G$ is the abelian group $\left(\mathbb{Z}_{p^{n}},+\right)$ and $\Phi$ is a proper non trivial subgroup of $A u t(G)$, and giving a rule for a multiplication " $*$ " on $\left(\mathbb{Z}_{p^{n}},+\right)$ which makes $\left(\mathbb{Z}_{p^{n}},+, *\right)$ a wd-nearring. In [5], starting from the wd-nearrings of $[3,4]$, a family of $P B I B D$ s ( $w d$-designs in the sequel) is constructed when $p$ is odd. As we are interested in the above wd-designs, in the following paragraph we summarize the main steps of the construction given in $[3,4,5]$.

\subsubsection{Construction}

First step. Consider the additive group $G=\left(\mathbb{Z}_{p^{n}},+\right), n>1$ and $p$ prime odd, and choose $\Phi$, a proper non trivial subgroup of $A u t(G)$ of order $t p^{h},(t, p)=1$. Set $Q=p \mathbb{Z}_{p^{n}}$ and $C=\mathbb{Z}_{p^{n}} \backslash Q$, so $N=C \cup Q$ and $C \cap Q=\emptyset$.

Select a set $E$ of the representatives $e_{x}$ of the $\Phi$-orbits covering $C$ in such a way that "if $e_{a}-e_{b} \notin$ $p^{j} \mathbb{Z}_{p^{n}},(j<n)$, then $x-y \notin p^{j} \mathbb{Z}_{p^{n}}, \forall x \in \Phi(a), \forall y \in \Phi(b)$ ". Fix $e$ among the elements of $E$ and 
define $^{1}$ :

$$
a * b= \begin{cases}0 & \text { if } a=0 \\ b p^{r} \phi_{k e^{r}}\left(e^{-r}\right) & \text { if } a=k p^{r} \text { with } k \in \mathbb{Z},(k, p)=1 \text { and } 0 \leq r<n\end{cases}
$$

Then $N=\left(Z_{p^{n}},+, *\right)$ results in a wd-nearring.

Second step. $\quad$ Now, both the nearring multiplication "*" and the usual ring multiplication (no symbol) are defined on $\mathbb{Z}_{p^{n}}$. Obviously $a * b=b(a * 1)$, for all $a, b \in \mathbb{Z}_{p^{n}}$. In the sequel, without loss of generality, we can set $e=1$. Thus we obtain $N * 1=\{0\} \cup \Phi(1) \cup \Phi(p) \cup \cdots \cup \Phi\left(p^{n-1}\right)$ and we deduce $N * a=a(N * 1)$.

In this way cyclic wd-designs can be generated:

orbital wd-designs $\mathcal{D}_{a}=\left(N, \mathcal{B}_{a}\right)$, where $a$ is a fixed element of $C$ and $\left.\mathcal{B}_{a}=\{N * a+b \mid b \in N)\right\}$. We obtain $c=p^{n-h-1}(p-1) / t$ orbital wd-designs, isomorphic to each other;

the union wd-design $\mathcal{D}=(N, \mathcal{B})$, where $\mathcal{B}=\{N * a+b \mid a \in C, b \in N\}=\bigcup_{a \in C} \mathcal{B}_{a}$. It is the union of the previous ones.

Third step. Consider the $\Phi$-orbit $\Delta_{i}$ and set $U_{i}=\Delta_{i} \cup\left(-\Delta_{i}\right)$. If $\Delta_{i}=-\Delta_{i}$, we say that $\Delta_{i}$ is self-paired and in this case $U_{i}=\Delta_{i}$. If $\Delta_{i}$ is not self-paired, then $\Delta_{i} \cap\left(-\Delta_{i}\right)$ is empty. Anyway we define $x$ and $y$ to be $i$ th associates if $x-y$ belongs to $U_{i}$. In this way an association scheme is given on $\left(Z_{p^{n}},+\right)$. It fits with the previously constructed block designs and we obtain PBIBDs.

\subsubsection{Notations and parameters}

In the sequel, to remember easily and quickly the main definitions and properties from $[3,4,5]$, the following keys could be useful.

\begin{tabular}{|c|c|c|}
\hline \multicolumn{3}{|c|}{ notations } \\
\hline$\Phi$ & automorphism group of $\left(\mathbb{Z}_{p^{n}},+\right)$ & $\begin{array}{c}1<|\Phi|=t p^{h}<(p-1) p^{n-1} \\
(t, p)=1\end{array}$ \\
\hline $\begin{array}{c}N= \\
\left(\mathbb{Z}_{\left.p^{n},+, *\right)}\right.\end{array}$ & constructed wd-nearring & $\begin{array}{c}N=C \cup Q \\
Q=p \mathbb{Z}_{p^{n}}, C=N \backslash Q\end{array}$ \\
\hline 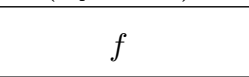 & number of the non trivial $\Phi$-orbits & $=\frac{(p h-h+p) p^{n-h-1}-1}{t}$ \\
\hline$c$ & number of the $\Phi$-orbits in $C$ & $=\frac{p-1}{t} p^{n-h-1}$ \\
\hline$E=\left(e_{j}\right)$ & representatives of the $\Phi$-orbits in $C$ & $j=1, \ldots, c$ \\
\hline$\Delta_{l}$ & $l$ th non trivial $\Phi$-orbit & $l=1, \ldots, f$ \\
\hline$\Delta_{i},-\Delta_{i}$ & paired $\Phi$-orbits & $\begin{array}{l}|\Phi| \text { even : self-paired } \\
|\Phi| \text { odd : not self-paired }\end{array}$ \\
\hline $\begin{array}{c}U_{i}= \\
\Delta_{i} \cup\left(-\Delta_{i}\right)\end{array}$ & union set of paired $\Phi$-orbits & $\begin{array}{ll}i=1, \ldots, m & \text { where } \\
m=f & \text { if }|\Phi| \text { is even } \\
m=f / 2 & \text { if }|\Phi| \text { is odd }\end{array}$ \\
\hline$D=\left(d_{i}\right)$ & representatives of the $U_{i}$ & $i=1, \ldots, m$ \\
\hline$B_{a, b}$ & block generated by $a \in C$ and $b \in N$ & $B_{a, b}=N * a+b$ \\
\hline$\left[B_{a_{1}, b_{1}}-B_{a_{2}, b_{2}}\right]$ & \multicolumn{2}{|c|}{$\begin{array}{l}\text { list of differences } \\
\text { between the elements of } B_{a_{1}, b_{1}} \text { and those of } B_{a_{2}, b_{2}}\end{array}$} \\
\hline$f_{a_{1}, a_{2}, k}$ & frequency of $k$ in $\left[B_{a_{1}, 0}-B_{a_{2}, 0}\right]$ & in particular $f_{a, a, k}=f_{a, k}$ \\
\hline $\mathcal{D}_{a}=\left(N, \mathcal{B}_{a}\right)$ & orbital wd-design & $\begin{array}{l}a \text { fixed element of } C \\
\left.\mathcal{B}_{a}=\left\{B_{a, b} \mid b \in N\right)\right\}\end{array}$ \\
\hline $\mathcal{D}=(N, \mathcal{B})$ & union wd-design & $\begin{array}{l}\mathcal{B}=\bigcup_{a \in C} \mathcal{B}_{a}= \\
\left\{B_{a, b} \mid a \in C, b \in N\right\}\end{array}$ \\
\hline
\end{tabular}

\footnotetext{
${ }^{1}$ We recall that $\phi_{x}$ denotes the automorphism of $\Phi$ such that $\phi_{x}\left(e_{x}\right)=x$, where $e_{x}$ is the selected representative of the orbit $\Phi(x)$.
} 


\begin{tabular}{|c|c|c|c|c|c|}
\hline \multicolumn{6}{|c|}{ parameters } \\
\hline \multicolumn{6}{|c|}{ association scheme } \\
\hline$x, y \in \mathcal{R}_{i}$ & \multicolumn{3}{|c|}{$i$ th associate elements } & \multicolumn{2}{|c|}{ if $x-y \in U_{i}$} \\
\hline$m$ & \multicolumn{3}{|c|}{ number of the associate classes } & \multicolumn{2}{|c|}{$\begin{array}{ll}m=f & \text { if }|\Phi| \text { is even } \\
m=f / 2 & \text { if }|\Phi| \text { is odd }\end{array}$} \\
\hline$n_{i}$ & \multicolumn{3}{|c|}{ number of the $i$ th associates elements } & \multicolumn{2}{|c|}{$\begin{array}{ll}n_{i}=\left|\Delta_{i}\right| & \text { if }|\Phi| \text { is even } \\
n_{i}=2\left|\Delta_{i}\right| & \text { if }|\Phi| \text { is odd }\end{array}$} \\
\hline \multirow[t]{2}{*}{$p_{i j}^{k}$} & \multicolumn{5}{|c|}{$\begin{array}{l}\text { number of the } i \text { th associates of } x \text { and } j \text { th associates of } y \\
\text { when } x \text { and } y \text { are } k \text { th associates }\end{array}$} \\
\hline & \multicolumn{4}{|c|}{ tactical configuration } & partial balance \\
\hline design & $v$ & $b$ & $k$ & $r$ & $\lambda_{i}$ \\
\hline $\mathcal{D}_{a}=\left(N, \mathcal{B}_{a}\right)$ & $p^{n}$ & $p^{n}$ & $\frac{p^{h+1}-1}{p-1} t+(n-h-1) t+1$ & $k$ & $\left(\lambda_{i}\right)_{a}=f_{a, d_{i}}=f_{1, d_{i} a^{-1}}$ \\
\hline $\mathcal{D}=(N, \mathcal{B})$ & $p^{n}$ & $c p^{n}$ & $\frac{p^{h+1}-1}{p-1} t+(n-h-1) t+1$ & $c k$ & $\lambda_{i}=\sum_{e_{j} \in E} f_{1, d_{i} e_{j}}$ \\
\hline
\end{tabular}

Propositions 4.1 and 4.2 of [5] show that, for any $k \in N$,

$$
f_{a_{1}, a_{2}, k}=f_{a_{1}, a_{2}, \phi(k)} \forall \phi \in \Phi, \quad \forall a_{1}, a_{2} \in C \quad \text { and } \quad f_{a, k}=f_{1, k a^{-1}}, \forall a \in C
$$

Thus, to know the frequency of $k$ in the list $\left[B_{a_{1}, 0}-B_{a_{2}, 0}\right]$ it is sufficient to know the frequency of any element of its orbit $\Phi(k)$ in the same list. Moreover, for any $a \in C$, the frequency of $k$ in $\left[B_{a, 0}-B_{a, 0}\right]$ equals the frequency of $k a^{-1}$ in $\left[B_{1,0}-B_{1,0}\right]$.

Previous results can be generalized by the following statements, in order to give some information about the cardinality of the block intersection.

Proposition 2.4 Let $B_{a_{1}, b_{1}}$ and $B_{a_{2}, b_{2}}$ be two blocks of the wd-design $\mathcal{D}=(N, \mathcal{B})$. Set $a=a_{2} a_{1}^{-1}$ and $k=\left(b_{2}-b_{1}\right) a_{1}^{-1}$. Then

$$
\left|B_{a_{1}, b_{1}} \cap B_{a_{2}, b_{2}}\right|=f_{1, a, k}
$$

Let $y \in B_{a_{1}, b_{1}} \cap B_{a_{2}, b_{2}}$. Then there are $x, \bar{x} \in N$ so that $y=x * a_{1}+b_{1}=\bar{x} * a_{2}+b_{2}$. Setting $a=a_{2} a_{1}^{-1}$ and $k=\left(b_{2}-b_{1}\right) a_{1}^{-1}$, we obtain $x * 1-\bar{x} * a=k$, thus $k \in\left[B_{1,0}-B_{a, 0}\right]$. Suppose that $y^{\prime}=x^{\prime} * a_{1}+b_{1}=\bar{x}^{\prime} * a_{2}+b_{2}$ belongs to $B_{a_{1}, b_{1}} \cap B_{a_{2}, b_{2}}$, then $x^{\prime} * 1-\bar{x}^{\prime} * a=k$, also. If $y^{\prime} \neq y$ we have $x * a_{1} \neq x^{\prime} * a_{1}$ and this implies $x * 1 \neq x^{\prime} * 1$. So, two different elements in $B_{a_{1}, b_{1}} \cap B_{a_{2}, b_{2}}$ produce two different occurrences of $k$ in $\left[B_{1,0}-B_{a, 0}\right]$. Conversely, if $k$ occurs in $\left[B_{1,0}-B_{a, 0}\right]$, we have $k=x^{\prime \prime} * 1-\bar{x}^{\prime \prime} * a$ for $a=a_{2} a_{1}^{-1}, k=\left(b_{2}-b_{1}\right) a_{1}^{-1}$ and for some $x^{\prime \prime}, \bar{x}^{\prime \prime} \in N$. Thus, there exists $y^{\prime \prime}=x^{\prime \prime} * a_{1}+b_{1}=\bar{x}^{\prime \prime} * a_{2}+b_{2}$ belonging to $B_{a_{1}, b_{1}} \cap B_{a_{2}, b_{2}}$.

Now, to prove the following proposition, let $O$ be a set of representatives of the $\Phi$-orbits and remember that $E$ is a set of representatives of the $\Phi$-orbits covering $C$.

Proposition 2.5 Let $\mathcal{D}=(N, \mathcal{B})$ be a wd-design. Let $B_{a_{1}, b_{1}}$ and $B_{a_{2}, b_{2}}$ be any two different blocks. Then

$$
\begin{aligned}
\max \left\{\left|B_{a_{1}, b_{1}} \cap B_{a_{2}, b_{2}}\right|, a_{1}, a_{2}\right. & \left.\in C, b_{1}, b_{2} \in N\right\}= \\
& =\max \left\{f_{1, a, k},(a, k) \in(E \times O) \backslash\{(1,0)\}\right\}
\end{aligned}
$$

From Proposition 2.4 we know that $\left|B_{a_{1}, b_{1}} \cap B_{a_{2}, b_{2}}\right|=f_{1, a_{2} a_{1}^{-1},\left(b_{2}-b_{1}\right) a_{1}^{-1}}$. When $a_{1}, a_{2}$ run over $C$, and $b_{1}, b_{2}$ run over $N, a_{2} a_{1}^{-1}$ gives us all the elements of $C$ and $\left(b_{2}-b_{1}\right) a_{1}^{-1}$ gives us all the 
elements of $N$. Moreover, we know that $N * a=N * \phi(a)$ and $f_{a_{1}, a_{2}, k}=f_{a_{1}, a_{2}, \phi(k)}$ for all $\phi \in \Phi$. So, to compute $\max \left\{f_{1, a, k}, a \in C, k \in N\right\}$, we can confine $a$ in $E$ and $k$ in $O$. Obviously, the case $(a=1, k=0)$ must be excluded because it would be to say that the two blocks coincide.

Observation 2.6 Theorem 4.5 of [5] tells us something about the $p_{i j}^{k}$. Precisely, if two of $U_{i}$, $U_{j}$ and $U_{k}$ are contained in a proper subgroup $N^{\prime}$ of $\left(\mathbb{Z}_{p^{n}},+\right)$ and the third one has an empty intersection with $N^{\prime}$, then $p_{i j}^{k}=p_{k j}^{i}=p_{i k}^{j}=0$. To compute the non zero $p_{i j}^{k}$ as well, we can also say that $p_{i j}^{k}$ equals the frequency of $d_{k}$, the representative of $U_{k}$, in the list $\left[U_{i}-U_{j}\right]$ of the differences between the element of $U_{i}$ and those of $U_{j}$.

\section{Incidence matrix of a wd-design}

If $\mathcal{B}=\left\{B_{1}, \ldots, B_{b}\right\}$ is a block design on $X=\left\{x_{1}, \ldots, x_{v}\right\}$ of parameters $(v, b, r, k)$, the $v \times b$ matrix $A=\left(a_{y z}\right)$ is called incidence matrix of the design when $a_{y z}=1$ if $x_{y} \in B_{z}$ and $a_{y z}=0$ if $x_{y} \notin B_{z}$. Here we remember that a matrix of the form $\left(\begin{array}{cccc}c_{0} & c_{1} & \ldots & c_{n-1} \\ c_{n-1} & c_{0} & \ldots & c_{n-2} \\ \vdots & \vdots & \ldots & \vdots \\ c_{1} & c_{2} & \ldots & c_{0}\end{array}\right)$ is called circulant matrix and, for a given circulant matrix $C$, we have

$$
\operatorname{det}(C)=\prod_{j=1}^{n} f\left(\epsilon_{j}\right) \quad \text { where } \quad f(x)=\sum_{z=0}^{n-1} c_{z} x^{z}
$$

is the defining polinomial of $C$ and $\epsilon_{1}, \epsilon_{2}, \ldots, \epsilon_{n}$ denote the distinct $n$th roots of unity, (see $[9,11]$ ).

Now, we come back to the wd-designs previously constructed. We know that, for every choice of $\mathbb{Z}_{p^{n}}$ and $\Phi \subseteq \operatorname{Aut}\left(\mathbb{Z}_{p^{n}},+\right)$, our wd-designs are partially balanced with respect to the same association scheme with $m$ associate classes, where either $m=f$, when $|\Phi|$ is even, or $m=f / 2$, when $|\Phi|$ is odd, $f$ being the number of the non trivial $\Phi$-orbits.

For convenience, in what follows each element of $\mathbb{Z}_{p^{n}}$ will be denoted via his smallest non negative representative, that is $\mathbb{Z}_{p^{n}}=\left\{0,1, \ldots, p^{n}-1\right\}$.

Let $E=\left\{e_{1}, e_{2}, \ldots, e_{c}\right\}$ be a set of representatives of the $\Phi$-orbits covering $C$. Consider the orbital wd-design $\mathcal{D}_{e_{s}}=\left(N, \mathcal{B}_{e_{s}}\right)$ and set $B_{e_{s}, z}=N * e_{s}+z$, as usual. Let $A_{s}=\left(a_{y z}\right)$ be the $p^{n} \times p^{n}$ incidence matrix of $\mathcal{D}_{e_{s}}$, where $a_{y z}=1$ if, and only if, $y-1 \in B_{e_{s}, z-1}$ and $a_{y z}=0$ otherwise, for $y, z=1, \ldots, p^{n}$. The incidence matrix of the union wd-design $\mathcal{D}=\left(N, \mathcal{B}=\bigcup_{e_{s} \in E} \mathcal{B}_{e_{s}}\right)$, is a $p^{n} \times c p^{n}$ matrix obtained by the juxtaposition of the $A_{s} s$, that is $A=\left(A_{1} A_{2} \ldots A_{c}\right)$.

Theorem 3.1 Let $\mathcal{D}_{e_{s}}=\left(N, \mathcal{B}_{e_{s}}\right)$ be an orbital wd-design with $m$ associate classes and $A_{s}$ its incidence matrix. Then, for $s=1, \ldots, c$

1. $A_{s}$ is a circulant matrix, symmetric if, and only if, $|\Phi|$ is even;

2. $\operatorname{det}\left(A_{s}\right)=k \prod_{j=1}^{p^{n}-1}\left(\sum_{z \in B_{1,0}} \epsilon^{j z}\right)$

3. $H_{s}=A_{s} \cdot A_{s}^{T}$ is a symmetric circulant matrix and $\operatorname{det}\left(H_{s}\right)=\prod_{j=1}^{p^{n}}\left(k+\sum_{i=1}^{m}\left(\lambda_{i}\right)_{s} \sum_{z \in U_{i}} \epsilon^{j z}\right)$ where $\epsilon$ is a primitive $p^{n}$ th root of unity and the $\left(\lambda_{i}\right)_{s}$ s are the partial balance parameters of the $\mathcal{D}_{e_{s}} s$. 
1. $A_{s}$ is circulant because $y-1 \in B_{e_{s}, z-1}$ if, and only if, $y \in B_{e_{s}, z}$, for $y, z=1, \ldots, p^{n}$. This implies $a_{y z}=a_{y+1} z+1$, subscripts $\bmod p^{n}$, for all $y, z=1, \ldots, p^{n}$. Now, let $|\Phi|$ be even. If $a_{y, 1}=1$, that is $y-1 \in B_{e_{s}, 0}$, it results in $-(y-1) \in B_{e_{s}, 0}$, because all the orbits are self paired. Hence $0 \in B_{e_{s}, y-1}$, that is $a_{1, y}=1$. The converse is analogous, thus $a_{y, 1}=a_{1, y}$ for $y=1, \ldots, p^{n}$. Using this last statement in addition to the circulant definition, when $y>z$ we have $a_{y z}=a_{y-(z-1), z-(z-1)}=a_{y-z+1,1}=a_{1, y-z+1}=a_{1+(z-1), y-z+1+(z-1)}=a_{z y}$, so we can conclude that $A_{s}$ is symmetric.

2. Setting $a_{1, z}=c_{z-1}$ and applying (3.1), for $s=1, \ldots, c$ we have

$$
\operatorname{det}\left(A_{s}\right)=\prod_{j=1}^{p^{n}}\left(\sum_{z=0}^{p^{n}-1} c_{z} \epsilon_{j}^{z}\right)=\prod_{j=1}^{p^{n}}\left(\sum_{z=0}^{p^{n}-1} c_{z} \epsilon^{j z}\right)
$$

where $\epsilon$ is a primitive $p^{n}$ th root of unity and we set $\epsilon_{j}=\epsilon^{j}$. We know that $c_{z}=a_{1, z+1}=1$ if, and only if, $0 \in B_{e_{s}, z}$ and this happens if, and only if, $-z \in B_{e_{s}, 0}$, for $z=0, \ldots, p^{n}-1$.

If $|\Phi|$ is even, $-z \in B_{e_{s}, 0}$ if, and only if, $z$ itself belongs to $B_{e_{s}, 0}$, because the $\Phi$-orbits are self paired. So $c_{z}=1 \Longleftrightarrow z \in B_{e_{s}, 0}$.

If $|\Phi|$ is odd, $-z \in B_{e_{s}, 0}$ if, and only if, $z \in-B_{e_{s}, 0}=\bar{B}_{e_{s}, 0}$, the block containing all the orbits paired to the orbits of $B_{e_{s}, 0}$. So $c_{z}=1 \Longleftrightarrow z \in \bar{B}_{e_{s}, 0}$.

Thus, applying (3.2), for $s=1, \ldots, c$ we have

$$
\begin{aligned}
& \operatorname{det}\left(A_{s}\right)=\prod_{j=1}^{p^{n}}\left(\sum_{z \in B_{e_{s}, 0}} \epsilon^{j z}\right)=\prod_{j=1}^{p^{n}}\left(\sum_{z \in j B_{e_{s}, 0}} \epsilon^{z}\right) \quad \text { when }|\Phi| \text { is even } \\
& \operatorname{det}\left(A_{s}\right)=\prod_{j=1}^{p^{n}}\left(\sum_{z \in \bar{B}_{e_{s}, 0}} \epsilon^{j z}\right)=\prod_{j=1}^{p^{n}}\left(\sum_{z \in j \bar{B}_{e_{s}, 0}} \epsilon^{z}\right) \quad \text { when }|\Phi| \text { is odd }
\end{aligned}
$$

where, obviously, $\bar{B}_{e_{s}, 0}=B_{e_{t}, 0}$, for some $t \in(1, \ldots, c)$.

Finally, for every $s=1, \ldots, c$, we can see that $\left(B_{e_{s}, 0}, 2 B_{e_{s}, 0}, \ldots, p^{n} B_{e_{s}, 0}\right)$ becomes $\left(e_{s}(N * 1), 2 e_{s}(N *\right.$ $\left.1), \ldots, p^{n} e_{s}(N * 1)\right)$, being $B_{e_{s}, 0}=e_{s}(N * 1)$. Since $\left\{e_{s}, 2 e_{s}, \ldots, p^{n} e_{s}\right\}=\mathbb{Z}_{p^{n}}$, rearranging the previous sequence we obtain $\left((N * 1), 2(N * 1), \ldots, p^{n}(N * 1)\right)$. Thus, $\forall s=1, \ldots, c$,

$$
\operatorname{det}\left(A_{s}\right)=\prod_{j=1}^{p^{n}}\left(\sum_{z \in B_{1,0}} \epsilon^{j z}\right)=k \prod_{j=1}^{p^{n}-1}\left(\sum_{z \in B_{1,0}} \epsilon^{j z}\right)
$$

being $\sum_{z \in B_{1,0}} \epsilon^{p^{n} z}=\left|B_{1,0}\right|=k$.

3. The matrix $H_{s}=A_{s} \cdot A_{s}^{T}=\left(h_{y z}\right)$ is obviously symmetric and circulant. The element $h_{y z}$ give us the number of the blocks of $\mathcal{D}_{e_{s}}$ containing both the elements $y-1$ and $z-1$, hence $h_{y y}=k$, for $y=1, \ldots, p^{n}$. When $y \neq z, y-1$ and $z-1$ are $i$-associates if, and only if, their difference belongs to $U_{i}$. So, $z \in U_{i}$ implies $h_{1, z+1}=\left(\lambda_{i}\right)_{s}$, for $i=1, \ldots, m$. Applying again (3.1), where $c_{z}=h_{1, z+1}$, we can write

$$
\operatorname{det}\left(H_{s}\right)=\prod_{j=1}^{p^{n}}\left(\sum_{z=0}^{p^{n}-1} c_{z} \epsilon^{j z}\right)=\prod_{j=1}^{p^{n}}\left(k+\sum_{i=1}^{m}\left(\lambda_{i}\right)_{s} \sum_{z \in U_{i}} \epsilon^{j z}\right)
$$

being $c_{0}=k$ and $c_{z}=\left(\lambda_{i}\right)_{s}$ when $z \in U_{i}$, for $i=1, \ldots, m$. 
Theorem 3.2 Let $\mathcal{D}=(N, \mathcal{B})$ be a union wd-design with $m$ associate classes and $A=\left(A_{1} A_{2} \ldots A_{c}\right)$ its incidence matrix. Then $H=A \cdot A^{T}$ is a symmetric circulant matrix and

$$
\operatorname{det}(H)=\prod_{j=1}^{p^{n}}\left(r+\sum_{i=1}^{m} \lambda_{i} \sum_{z \in U_{i}} \epsilon^{j z}\right)
$$

where the $\lambda_{i}$ s are the partial balance parameters of $\mathcal{D}=(N, \mathcal{B})$.

$H=A \cdot A^{T}$ is circulant because it is a sum of circulant matrices. The statement follows similarly to the point 3. of previous Theorem 3.1, but now $c_{0}=\underbrace{k+\cdots+k}_{c \text { times }}=c k=r$ and $c_{z}=\lambda_{i}=\sum_{s=1}^{c}\left(\lambda_{i}\right)_{s}$ when $z \in U_{i}$, for $i=1, \ldots, m$.

\section{Row and column codes from $P B I B D$ s}

A binary code of length $n$ is a subset $C$ of $\mathbb{Z}_{2}^{n}$. The weight of a codeword is the number of its non zero coordinate places. The Hamming distance between two codewords is the number of coordinate places in which they differ. The smallest of the distances between distinct codewords is called minimum distance of $C$ and denoted by $d(C)$. A binary code of length $n$ having $m$ codewords and minimum distance $d$ is called a binary $(n, m, d)$-code. If $C$ is a vector subspace of $\mathbb{Z}_{2}^{n}$ and $\operatorname{dim}(C)=k$, then it is called a binary linear $(n, k)$-code.

It is well known that there is a link between block designs and codes via the design incidence matrix $A$ : the set of all the columns, the set of all the rows of $A$, as well as their linear hulls can be regarded as binary codes and $A$ itself can be regarded as the parity check matrix of a linear code. For more information on codes see $[1,12]$.

In what follows we are interested in the set $C_{c}$ of all the columns of $A$ and the set $C_{r}$ of all the rows of $A$, the so called column code and row code, respectively. The parameters characterizing $C_{c}$ and $C_{r}$ depend on the design parameters, as we summarize in the following proposition.

Proposition 4.1 Let $\mathcal{B}$ be a PBIBD with parameters $\left(v, b, r, k, \lambda_{1}, \ldots, \lambda_{m}\right)$, $A$ its incidence matrix, $\mathcal{C}_{r}$ and $\mathcal{C}_{c}$ the related row and column codes. Set $\lambda=\max \left\{\lambda_{1}, \ldots, \lambda_{m}\right\}$ and $\mu=\max \left\{\mid B_{i} \cap\right.$ $B_{j} \mid, i, j=1, \ldots, b \quad$ with $\left.i \neq j\right\}$. Then

(1) the cardinality of $\mathcal{C}_{r}$ is $v$, the codeword length is b, each codeword has the same weight $r$ and the minimum distance is $d\left(\mathcal{C}_{r}\right)=2(r-\lambda)$.

(2) the cardinality of $\mathcal{C}_{c}$ is $b$, the codewords length is $v$, each codeword has the same weight $k$ and the minimum distance is $d\left(\mathcal{C}_{c}\right)=2(k-\mu)$.

\subsection{Row and column codes from wd-designs}

Now, we come back to the PBIBDs of [5], the so called wd-designs recalled in Paragraph 2, and we consider their incidence matrices. Working on $\mathbb{Z}_{p^{n}}$ with $|\Phi|=t p^{h}$, each orbital design has a $p^{n} \times p^{n}$ matrix $A_{i}$, for $i=1, \ldots, c$, and $A=\left(A_{1} \ldots A_{c}\right)$ is the incidence matrix of the union block design. We can see that, applying previous Propositions and using the keys of Paragraph 2.1.2, we are able to compute all the wd-design parameters as well as those of the row and column codes related to their incidence matrices and we obtain: 


\begin{tabular}{|c|c|c|c|c|c|}
\hline \multicolumn{7}{|c|}{ row and column codes } \\
\hline design & code & words & length & $\mathrm{d}$ & weight \\
\hline $\mathcal{D}_{a}=\left(N, \mathcal{B}_{a}\right)$ & $C_{r}$ or $C_{c}$ & $p^{n}$ & $p^{n}$ & $2\left(k-\lambda_{a}\right)$ & $k$ \\
\hline $\mathcal{D}=(N, \mathcal{B})$ & $C_{r}$ & $p^{n}$ & $c p^{n}$ & $2(c k-\lambda)$ & $c k$ \\
\hline & $C_{c}$ & $c p^{n}$ & $p^{n}$ & $2(k-\mu)$ & $k$ \\
\hline
\end{tabular}

where

$$
\begin{aligned}
& k=\frac{p^{h+1}-1}{p-1} t+(n-h-1) t+1, c=\frac{p-1}{t} p^{n-h-1}, \lambda_{a}=\max \left\{f_{1, d_{i} a^{-1}}, d_{i} \in D\right\}, \\
& \lambda=\max \left\{\sum_{e_{j} \in E} f_{1, d_{i} e_{j}}, d_{i} \in D\right\}, \mu=\max \left\{f_{1, a, k}, \quad(a, k) \in(E \times O) \backslash\{(1,0)\}\right\} .
\end{aligned}
$$

\section{Some special classes of wd-designs}

From the key of page 4 we know that the number $m$ of the associate classes of a wd-design is $f$ or $f / 2$, according to the order of $\Phi$ is even or odd.

Thus, if we want a given number of associate classes, we have to compute case by case. For example, if we want an eight-class wd-design, we can work on $\mathbb{Z}_{3^{8}}$ with $|\Phi|=3^{7}$ as well as on $\mathbb{Z}_{5^{4}}$ with $|\Phi|=2.5^{3}$ as well as on $\mathbb{Z}_{13^{4}}$ with $|\Phi|=3.13^{3}$ and so on.

Now, two special cases will be examined, for which the previous formulae can be developed further.

\subsection{The case with $|\Phi|=p^{n-1}$}

We are following the line described in Section 2, Paragraph 2.1.

First step. In $\operatorname{Aut}\left(\mathbb{Z}_{p^{n}}\right)$ we choose $\Phi=<\alpha_{p+1}>^{2}$, thus $|\Phi|=p^{n-1}$. So we start from $p=p$, $n=n, h=n-1$ and $t=1$. Using the keys of Paragraph 2.1.2 we can compute the number $c$ of the $\Phi$-orbits covering $C=\mathbb{Z}_{p^{n}} \backslash p \mathbb{Z}_{p^{n}}: c=p-1$. Now we have to select a set $E$ of representatives of these $\Phi$-orbits and, for convenience, we want $1 \in E$. An easy selection is $E=\left(e_{j}\right)_{j \in I_{p-1}}=(1,2, \ldots, p-1)$. Now, from [4], we learn that a new multiplication " $*$ " can be defined on $\mathbb{Z}_{p^{n}}$ to obtain a wd-nearring $N=\left(\mathbb{Z}_{p^{n}},+, *\right)$ and we have $N * 1=\Phi(1) \cup \Phi(p) \cup \cdots \cup \Phi\left(p^{n-1}\right) \cup\{0\}$ (see Paragraph 2.1.1), where $\Phi\left(p^{s}\right)=\left\{p^{s}+h_{s+1} p^{s+1}, \quad 0 \leq h_{s+1}<p^{n-s-1}\right\}$, for $s=0, \ldots, n-1$.

Second step. Using the sets $N * a+b$, with $a \in C$ and $b \in N$, as blocks, wd-designs can be constructed. We have $p-1$ orbital wd-designs, generated by the $p-1$ basic blocks $N * 1, \ldots, N *(p-1)$ and isomorphic to each other, of parameters $v=b=p^{n}$ and $k=r=\left(p^{n}+p-2\right) /(p-1)$ and one union wd-design, union of the previous ones, of parameters $v=p^{n}, b=(p-1) p^{n}, k=$ $\left(p^{n}+p-2\right) /(p-1)$ and $r=p^{n}+p-2$.

Third step. Using the keys of Paragraph 2.1.2 we can compute the number of the non trivial $\Phi$-orbits, $f=n(p-1)$. As $|\Phi|$ is odd, we know that the non trivial $\Phi$-orbits are not self-paired, so we define the $U_{i} \mathrm{~s}$ by pairing them, that is $U_{i}=\Delta_{i} \cup-\Delta_{i}$, with $i=1, \ldots, f / 2$. Thus, we obtain an association scheme with $m=n(p-1) / 2$ associate classes defining $x$ and $y$ to be $i$ th associates when $x-y$ belongs to $U_{i}$. To compute the association scheme parameters, the $U_{i} \mathrm{~s}$ have to be ordered and we can do it by choosing $D$, a set of their representatives: $D=\left(d_{i}\right)_{i \in I_{m}}=$ $\left(1,2, \ldots, \frac{p-1}{2}, p, 2 p, \ldots, \frac{p-1}{2} p, \ldots, p^{n-1}, 2 p^{n-1} \ldots, \frac{p-1}{2} p^{n-1}\right)$. We know that the number of the $i$ th

\footnotetext{
${ }^{1}$ for $s \in \mathbb{Z}$ and $(s, p)=1, \quad \alpha_{s}$ is the automorphism of $\mathbb{Z}_{p^{n}}$ s. t. $\alpha_{s}(x)=s x, \forall x \in \mathbb{Z}_{p^{n}}$.
} 
associates of each element depends on $i$ only, and now we have $n_{i}=\left|U_{i}\right|$, thus $n_{i}=2 p^{n-j}$, where $j=1, \ldots, n$ and $i=(j-1)(p-1) / 2+1, \ldots, j(p-1) / 2$.

Since $m=n(p-1) / 2$, the $p_{i j}^{k}$ fill in $n(p-1) / 2$ squared matrices of order $n(p-1) / 2$ : the $P_{k} \mathrm{~s}$. Now, applying the first part of Observation 2.6, we obtain the general structure of these matrices. In fact, for $l=1, \ldots, n$, if one of $k, i$ or $j$ belongs to $\{(l-1)(p-1) / 2+1, \ldots, l(p-1) / 2\}$ and the two others are greater than $l(p-1) / 2$, we have $p_{i j}^{k}=p_{k j}^{i}=p_{i k}^{j}=0$. So, setting

$$
\begin{gathered}
Q_{k r}=\left(p_{i j}^{k}\right) \quad \text { with } i, j=(r-1) \frac{p-1}{2}+1, \ldots, r \frac{p-1}{2}, \quad r=1, \ldots, n \\
H_{k r+s}=\left(p_{i j}^{k}\right) \quad \text { with }\left\{\begin{array}{l}
i=(r-1) \frac{p-1}{2}+1, \ldots, r \frac{p-1}{2}, \quad r=1, \ldots, n \\
j=(r+s-1) \frac{p-1}{2}+1, \ldots,(r+s) \frac{p-1}{2} \quad s=1, \ldots, n-r
\end{array}\right. \\
\text { for } l=1, \ldots, n \quad \text { and } k=(l-1)(p-1) / 2+1, \ldots, l(p-1) / 2, \quad \text { we obtain }
\end{gathered}
$$

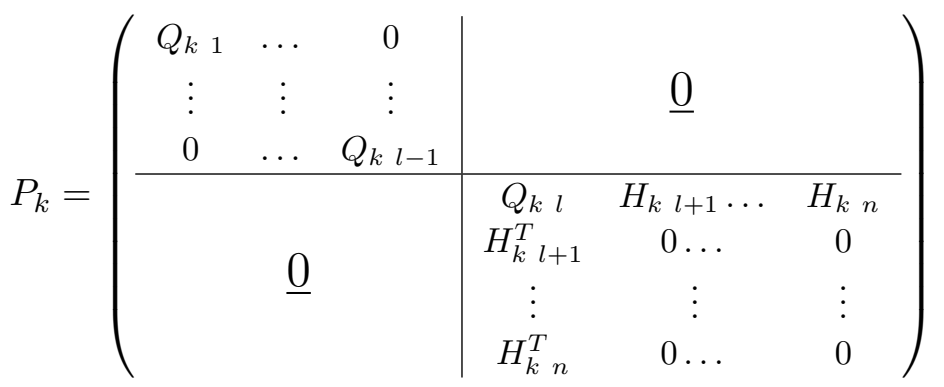

Fourth step. To compute the partial balance parameters applying the keys of Paragraph 2.1.2, we need the frequencies $f_{1, d_{i} e_{j}}$ for $d_{i} \in D$ and $e_{j} \in E$. Actually, it is sufficient to compute $f_{1,1}=1+1+p+\cdots+p^{n-2}=1+\frac{p^{n-1}-1}{p-1}, \quad f_{1,2}=\cdots=f_{1,(p-1) / 2}=0, \quad$ and, for $h=1, \ldots, n-1, \quad f_{1, p^{h}}=$ $p^{n-1}+p^{n-2}+\cdots+p^{n-h}+1+\frac{p^{n-h-1}-1}{p-1}, f_{1,2 p^{h}}=\cdots=f_{1, p^{h}(p-1) / 2}=p^{n-1}+p^{n-2}+\cdots+p^{n-h}$, to obtain

\begin{tabular}{|l|}
\hline for $\mathcal{D}_{1}$, the orbital wd-design generated by $B_{1,0}=N * 1$ with $\left(\lambda_{i}\right)_{1}=f_{1, d_{i} \cdot 1}=f_{1, d_{i}}$ \\
\hline for $h=0, \ldots, n-1$ \\
$\lambda_{h(p-1) / 2+1}=f_{1, p^{h}}=p^{n-1}+p^{n-2}+\cdots+p^{n-h}+1+\frac{p^{n-h-1}-1}{p-1}$ \\
$\lambda_{h(p-1) / 2+2}=f_{1,2 p^{h}}=\cdots=\lambda_{(h+1)(p-1) / 2}=f_{1, p^{h}(p-1) / 2}=p^{n-1}+p^{n-2}+\cdots+p^{n-h}$ \\
$\lambda=\max \left\{\lambda_{1}, \ldots, \lambda_{n(p-1) / 2}\right\}=\frac{p^{n}-1}{p-1}$ \\
\hline
\end{tabular}

For $\mathcal{D}_{2}, \ldots, \mathcal{D}_{p-1}$ we find the same values but, obviously, not in the same order.

\begin{tabular}{|l|}
\hline \multicolumn{1}{c|}{ for $\mathcal{D}$, the union wd-design } \\
\hline for $h=0, \ldots, n-1$ \\
$\lambda_{h(p-1) / 2+1}=\sum_{e_{j} \in E} f_{1, d_{h(p-1) / 2+1} e_{j}}=2\left(f_{1, p^{h}}+f_{1,2 p^{h}}+\cdots+f_{1, p^{h}(p-1) / 2}\right)=$ \\
$2+p^{n}-p^{n-h}+2 \frac{p^{n-h-1}-1}{p-1}=\lambda_{h(p-1) / 2+2}=\cdots=\lambda_{(h+1)(p-1) / 2}$ \\
$\lambda=\max \left\{\lambda_{1}, \ldots, \lambda_{n(p-1) / 2}\right\}=p^{n}-p+2$
\end{tabular}

There remains to compute $\mu$, the maximum cardinality of the block intersections. Obviously, for each orbital wd-design we have $\mu=\lambda$. For the union wd-design, applying Proposition 2.5, we have to compute the $f_{1, a, k} \mathrm{~s}$, for $(a, k) \in(E \times O) \backslash\{(1,0)\}$. $E$ was already defined as $(1,2, \ldots, p-1)$. $O$, a set of representatives of all the $\Phi$-orbits, can be taken as $O=E \cup p E \cup \cdots \cup p^{n-1} E \cup\{0\}$. After a straightforward computation we obtain again $\mu=\left(p^{n}-1\right) /(p-1)$. Thus, applying the key of Paragraph 4.1, for the row and column codes we have: 


\begin{tabular}{|c|c|c|c|c|c|}
\hline \multicolumn{7}{|c|}{ row and column codes } \\
\hline wd-design & code & words & length & d & weight \\
\hline $\mathcal{D}_{1}, \ldots, \mathcal{D}_{p-1}$ & $C_{r}$ or $C_{c}$ & $p^{n}$ & $p^{n}$ & 2 & $\left(p^{n}+p-2\right) /(p-1)$ \\
\hline $\mathcal{D}$ & $C_{r}$ & $p^{n}$ & $(p-1) p^{n}$ & $4(p-2)$ & $\left(p^{n}+p-2\right)$ \\
\hline & $C_{c}$ & $(p-1) p^{n}$ & $p^{n}$ & 2 & $\left(p^{n}+p-2\right) /(p-1)$ \\
\hline
\end{tabular}

Finally, applying Theorems 3.1 and 3.2, for the incidence matrix we obtain:

\begin{tabular}{|c|}
\hline incidence matrix $A=\left(A_{1} \ldots A_{p-1}\right)$ \\
\hline$\left|A_{1}\right|=\cdots=\left|A_{p-1}\right|=k \prod_{j=1}^{n-1}\left[\frac{p^{j p}+\left(p^{j-1}+\cdots+p+2\right)^{p}}{p^{j}+\cdots+p+2}\right]^{p^{n-j-1}}$ \\
$(p-1) k^{2} \prod_{h=0}^{n-1}\left[(p-1) k-p^{h} \lambda_{(n-h) \frac{p-1}{2}}+(p-1) \sum_{a=0}^{h-1} p^{a} \lambda_{(n-a) \frac{p-1}{2}}\right]^{p^{n-h-1}(p-1)}$ \\
\hline
\end{tabular}

\subsection{The case with $|\Phi|=\frac{p-1}{2} p^{n-1}$}

Following, as above, the line of Paragraph 2.1.1 of Section 2, if we work on $\mathbb{Z}_{p^{n}}$ and we choose $|\Phi|=\frac{p-1}{2} p^{n-1}$ with $p \equiv 3(\bmod 4)$, we obtain an already known class of PBIBDs. The union wddesign $\mathcal{D}=(N, \mathcal{B})$ can be split into two orbital wd-designs $\mathcal{D}_{1}=\left(N, \mathcal{B}_{1}\right)$, generated by $B_{1,0}=N * 1$, and $\mathcal{D}_{2}=\left(N, \mathcal{B}_{2}\right)$ generated by $B_{p^{n}-1,0}=N *\left(p^{n}-1\right)$. All the designs have the same association scheme with $n$ associate classes and they, their incidence matrices and the related row and column codes are described in the following tables:

\begin{tabular}{|c|l|l|}
\hline \multicolumn{3}{|c|}{ association scheme } \\
\hline$x, y \in \mathcal{R}_{i}$ & $i$ th associate elements, for $i=1, \ldots, n$ & if $x-y \in U_{i}=p^{i-1} \mathbb{Z}_{p^{n}} \backslash p^{i} \mathbb{Z}_{p^{n}}$ \\
\hline$m$ & number of the associate classes & $m=f / 2=n$ \\
\hline$n_{i}$ & number of the $i$ th associates elements & $n_{i}=(p-1) p^{n-i}$ for $i=1, \ldots, m$ \\
\hline
\end{tabular}

As usual we set $P_{k}=\left(p_{i j}^{k}\right)$ for $k=1, \ldots, m$

$$
P_{k}=\left(\begin{array}{cccccccc}
n_{1} & 0 & \ldots & 0 & 0 & 0 & \ldots & 0 \\
0 & n_{2} & \ldots & 0 & 0 & 0 & \ldots & 0 \\
\vdots & \vdots & & \vdots & \vdots & \vdots & & \vdots \\
0 & 0 & \ldots & n_{k-1} & 0 & 0 & \ldots & 0 \\
0 & 0 & \ldots & 0 & (p-2) p^{n-k} & n_{k+1} & \ldots & n_{m} \\
0 & 0 & \ldots & 0 & n_{k+1} & 0 & \ldots & 0 \\
\vdots & \vdots & & \vdots & \vdots & \vdots & & \vdots \\
0 & 0 & \ldots & 0 & n_{m} & 0 & \ldots & 0
\end{array}\right)
$$

\begin{tabular}{|c|c|c|c|c|c|}
\hline & \multicolumn{4}{|c|}{ tactical configuration } & partial balance \\
\hline design & $v$ & $b$ & $k$ & $r$ & $\lambda_{i}$ for $i=1, \ldots, n$ \\
\hline $\mathcal{D}_{1}$ or $\mathcal{D}_{2}$ & $p^{n}$ & $p^{n}$ & $\frac{p^{n}+1}{2}$ & $\frac{p^{n}+1}{2}$ & $\left(\lambda_{i}\right)_{1}=\left(\lambda_{i}\right)_{2}=\left(2 p^{n}-p^{n-i+1}-p^{n-i}+2\right) / 4$ \\
\hline $\mathcal{D}$ & $p^{n}$ & $c p^{n}$ & $\frac{p^{n}+1}{2}$ & $p^{n}+1$ & $\lambda_{i}=\left(2 p^{n}-p^{n-i+1}-p^{n-i}+2\right) / 2$ \\
\hline
\end{tabular}




\begin{tabular}{|l|l|l|}
\hline \multicolumn{3}{|c|}{ incidence matrix $A=\left(A_{1} A_{2}\right)$} \\
\hline \multicolumn{2}{|c|}{$A_{1}=A_{2}^{T} \quad A_{1}+A_{2}=J+I$} & $A * A^{T}=2\left(A_{1} * A_{1}^{T}\right)=2\left(A_{2}^{T} * A_{2}\right)$ \\
\hline \multirow{3}{*}{$A_{s}$} & incidence matrix of & $\left|A_{1}\right|=\left|A_{2}\right|=$ \\
& $\mathcal{D}_{s}=\left(N, \mathcal{B}_{s}\right) \quad s=1,2$ & $k \prod_{j=1}^{n}\left[\left(1+p^{2(n-j)+1}\right) / 4\right]^{p^{j-1}(p-1) / 2}$ \\
\hline \multirow{3}{*}{$A$} & \multirow{3}{*}{ incidence matrix of $\mathcal{D}=(N, \mathcal{B})$} & $\left|A \cdot{ }^{t} A\right|=$ \\
& & $2 k^{2} \prod_{j=1}^{n}\left[\left(1+p^{2(n-j)+1}\right) / 2\right]^{p^{j-1}(p-1)}$ \\
\hline
\end{tabular}

\begin{tabular}{|c|c|c|c|c|c|}
\hline \multicolumn{7}{|c|}{ row and column codes } \\
\hline design & code & words & length & d & weight \\
\hline $\mathcal{D}_{1}$ or $\mathcal{D}_{2}$ & $C_{r}$ or $C_{c}$ & $p^{n}$ & $p^{n}$ & $(p+1) / 2$ & $\left(p^{n}+1\right) / 2$ \\
\hline $\mathcal{D}$ & $C_{r}$ & $p^{n}$ & $2 p^{n}$ & $p+1$ & $p^{n}+1$ \\
\hline & $C_{c}$ & $2 p^{n}$ & $p^{n}$ & $(p+1) / 2$ & $\left(p^{n}+1\right) / 2$ \\
\hline
\end{tabular}

\section{References}

[1] Assmus Jr., E.F. and Key, J.D., Designs and their codes, Cambridge Tractas in Mathhematics, Cambridge University Press, NY 1992.

[2] Bannai, E., Introduction to association schemes, Methods of Discrete Mathematics, (Braunschweig,1999), 1-70.

[3] Benini, A. and Morini, F., Weakly divisible nearrings on the group of integers $\left(\bmod p^{n}\right)$, Riv. Mat. Univ. Parma, (6) 1 (1998), 1-11.

[4] Benini, A. and Morini, F., On the construction of a class of weakly divisible nearrings, Riv. Mat. Univ. Parma, (6) 1 (1998), 103-111.

[5] Benini, A. and Morini, F., Partially Balanced Incomplete Block Designs from Weakly Divisible Nearrings, Sem. Mat. Brescia, Quad. 17 (2004), preprint.

[6] Benini, A. and Pellegrini, S., Weakly Divisible Nearrings, Discrete Math. 208/209 (1999), 49-59.

[7] Beth, T., Jungnickel, D. and Lenz, H., Design Theory, Encyclopedia of Mathematics and its Applications, 69. Cambridge University Press, Cambridge 1999.

[8] Clay, J.R., Nearrings: Geneses and Applications, Oxford Science Publications, Oxford University Press, NY 1992.

[9] Davis, P.J., Circulant matrices, 2nd. ed., Chelsea Publishing, New York, NY 1994.

[10] Hall, M., Designs with transitive authomorphism group, Proc. of Symposia in Pure Math. AMS, T. L. Motzkin, ed., (1971), 109-113.

[11] Lancaster, P. and Timenetsky, M., The theory of matrices, 2nd. ed. ACADEMIC PRESS, 1985.

[12] McWilliams, F.J. and Sloane, N.J.A., The theory of Error-correcting Codes, North-Holland Amsterdam 1977.

[13] Penfold Street, A. and Street, D.J., Combinatorics of Experimental Design Oxford University Press, New York, 1987.

[14] Pilz, G., Near-rings, 2nd. ed., North Holland Math. Studies 23, Amsterdam, 1983.

Anna Benini,

Dipartimento di Matematica,

Facoltà di Ingegneria,

Università degli Studi di Brescia,

Via Valotti 9, I-25133 BRESCIA, Italy.

Email: anna.benini@ing.unibs.it

Eingegangen am 13. Dezember 2004 